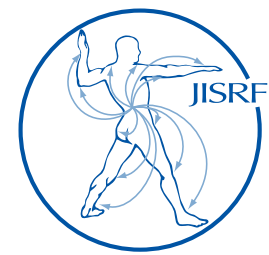

\title{
Leg and Femoral Neck Length Evaluation Using an Anterior Capsule Preservation Technique in Primary Direct Anterior Approach Total Hip Arthroplasty
}

\author{
Nelson $S^{1}$, Adrados $M^{1}$, Gala $R^{1}$, Geiger $E^{1}$, Webb $M^{1}$, Rubin $L^{1}$, Keggi $K^{1}$
}

\begin{abstract}
Background: Achieving correct leg and femoral neck lengths remains a challenge during total hip arthroplasty (THA). Several methods for intraoperative evaluation and restoration of leg length have been proposed, and each has inaccuracies and shortcomings. Both the supine positioning of a patient on the operating table during the direct anterior approach (DAA) THA and the preservation of the anterior capsule tissue are simple, readily available, and cost-effective strategies that can lend themselves well as potential solutions to this problem.

Technique: The joint replacement is performed through a longitudinal incision (capsulotomy) of the anterior hip joint capsule, and release of the capsular insertion from the femoral intertrochanteric line. As trial components of the prosthesis are placed, the position of the released distal capsule in relationship to its original insertion line is an excellent guide to leg length gained, lost, or left unchanged.

Methods: The radiographs of 80 consecutive primary THAs were reviewed which utilized anterior capsule preservation and direct capsular measurement as a means of assessing change in leg/femoral neck length. Preoperatively, the operative legs were $2.81+/-8.5 \mathrm{~mm}(\mathrm{SD})$ shorter than the nonoperative leg (range: $17.7 \mathrm{~mm}$ longer to $34.1 \mathrm{~mm}$ shorter). Postoperatively, the operative legs were $1.05+/-5.64 \mathrm{~mm}$ (SD) longer than the nonoperative leg (range: $14.9 \mathrm{~mm}$ longer to $13.7 \mathrm{~mm}$ shorter).

Conclusion: The preservation and re-assessment of the native anterior hip capsule in relationship to its point of release on the femur is a simple and effective means of determining leg/femoral neck length during DAA THA.
\end{abstract}

Keywords: total hip arthroplasty; leg length discrepancy; femoral neck length; direct anterior approach; hip capsule; capsulotomy; capsulectomy; capsulorrhaphy

Level of Evidence: AAOS Therapeutic Level IV

1 Stephen J Nelson MD, Murillo Adrados MD, Raj J Gala MD, Erik J Geiger MD, Matthew L Webb MD, Lee Rubin MD, Kristaps J Keggi MD

Department of Orthopaedics and Rehabilitation, Yale School of Medicine, 800 Howard Avenue, New Haven, CT, 06510, USA

(Direct reprint requests to Stephen J Nelson)

\footnotetext{
(C) 2017 Nelson, Adrados, Gala, Geiger, Webb, Rubin, Keggi. All rights reserved. Authors retain copyright and grant the journal right of first publication with the work. Reconstructive Review follows the Creative Commons Attribution-NonCommercial CC BY-NC. This license allows anyone to OPEN ACCESS download works, build upon the material, and share them with others for non-commercial purposes as long as they credit the senior author, Reconstructive Review, and the Joint Implant Surgery \& Research Foundation (JISRF). An example credit would be: "Courtesy of (senior author's name), Reconstructive Review, JISRF, Chagrin Falls, Ohio".
} 


\section{Introduction}

Maintaining both hip stability and appropriate leg lengths is one of the great challenges of total hip arthroplasty (THA). Incorrect leg length is a major cause of morbidity after THA and has been associated with back pain, sciatica, neuritis, gait disorders, general dissatisfaction, early loosening of components and revision surgery [1-7]. Additionally, patients who perceive leg length discrepancy have been found to have worse Oxford Hip scores. [ $\underline{8}, \underline{9}]$ With such morbidity, LLD is second only to nerve injury as the most common cause of litigation after THA [10]. As such, the potential for iatrogenic leg length discrepancy is a known risk of THA that should be discussed with patients before surgery and documented accordingly, explaining that a minor increase in leg length is not uncommon and perhaps preferable to a dislocated hip.

Preoperative assessment and implant templating are important considerations. Templating is an important guide to intraoperative decision-making, but excellent planning does not guarantee excellent execution [11]. Actual sizing of implanted components has been reported to match preoperative templating in only $60 \%$ of cases [12], and therefore should not be relied upon as the only means of determining leg or femoral neck length.

There have been over 20 intraoperative methods described for evaluating limb length and include the Ober test, the shuck test, and the drop kick test $[\underline{13}, 14]$. These methods utilize soft tissue tension with components in place as an indicator for limb length, but anesthesia induced muscle relaxation may limit the trustworthiness of these tests. Direct leg-to-leg comparison can also be useful but the palpation of anatomic landmarks may be inaccurate under surgical drapes, especially during lateral decubitus patient positioning for THA.

Fluoroscopically guided THA has increased in popularity recently, as it can ensure correct component position radiographically and may facilitate proper leg lengths. However, this method potentially adds operative time, requires both a radiolucent table and an image intensifier, and may increase required personnel and equipment traffic, all of which can carry an increased risk of wound contamination and surgical site infection. The additional radiation exposure to patient and staff is also a matter to consider [15].

Intraoperative navigation and length measurement techniques are usually based on 2 reference points marked on the pelvis and femur. This can be achieved via iliac fixation pins, intraoperative calipers, infracotyloid pins, or fixed suture lengths [16]. None of these methods are perfect, and many inconsistencies have been described [11].

Computer navigation and robot assisted surgery have also been developed to reconstruct normal anatomy and proper leg length. However, these methods are expensive, not widely available, and do not address the key issue of restoring soft tissue tension. For the present, they pale to the intraoperative judgement of an experienced surgeon.

The senior author and his colleagues have used the anterior, internervous, muscle-sparing surgical approach since 1973 , in some 15,000 primary and revision hip arthroplasties [17-19]. This anterior approach was presented as a scientific exhibit at the Annual Meeting of the American Academy of Orthopaedic Surgeons (AAOS) in 1977, and published in 1980 [17]. During the first 30 years of his work with the DAA, a total or subtotal capsulectomy was the norm. The results were satisfactory, but to more accurately restore leg length and attempt to further increase joint stability, a technique for anterior hip capsulotomy with anatomic capsule preservation, whenever possible, was evolved. It has helped to decrease soft tissue dissection, reduce the dead space created by a capsulectomy, and has the additional benefit of allowing for the intraoperative assessment of leg/femoral neck length, as is described within this paper.

\section{Surgical Technique}

With the patient supine on the operating table, a short oblique incision is made distal and lateral to the anterior superior iliac spine overlying the femoral neck. The medial border of the tensor fascia lata muscle is identified. The muscle and its fascia are then split longitudinally, approximately $1 \mathrm{~cm}$ lateral to its medial border. The medial strip of fascia and muscle can add to safety by protecting the small branches of the lateral femoral cutaneous nerve and the femoral neurovascular bundle. Cobra retractors are then placed on the superior and inferior aspects of the hip capsule. The superior Cobra retracts the tensor fascia lata and the abductors. The inferior Cobra retracts the rectus, sartorius, and iliopsoas. These two Cobras provide excellent exposure of the anterior hip capsule, which can then be further defined by the removal of its overlying fatty tissues.

An assessment is then made if further capsular visualization is necessary, which can be achieved by dissection with elevation and/or release of the reflected head of the rectus femoris by the proximal placement of a third blunt Cobra under its fibrous tendon with the tip of the Cobra in place over the anterior acetabulum with the tip just over the anterior rim of pelvis. In most cases of elderly patients without excessive acetabular or femoral head osteophytes and an atrophied reflected rectus, this third Cobra is not necessary and the capsule can be incised by starting the 
incision underneath the tendon, but the morphology of the reflected head can be larger and more muscular, particularly in younger males.

The exposed anterior capsule is incised in line with the long axis of the underlying femoral neck, and released from its distal lateral insertion on the intertrochanteric line (Figure 1). If necessary and if it is tight, the medial distal portion can also be released, creating an inverted T-capsulotomy. The two Cobras are then placed inside of the capsule on the superior and inferior portions of the femoral

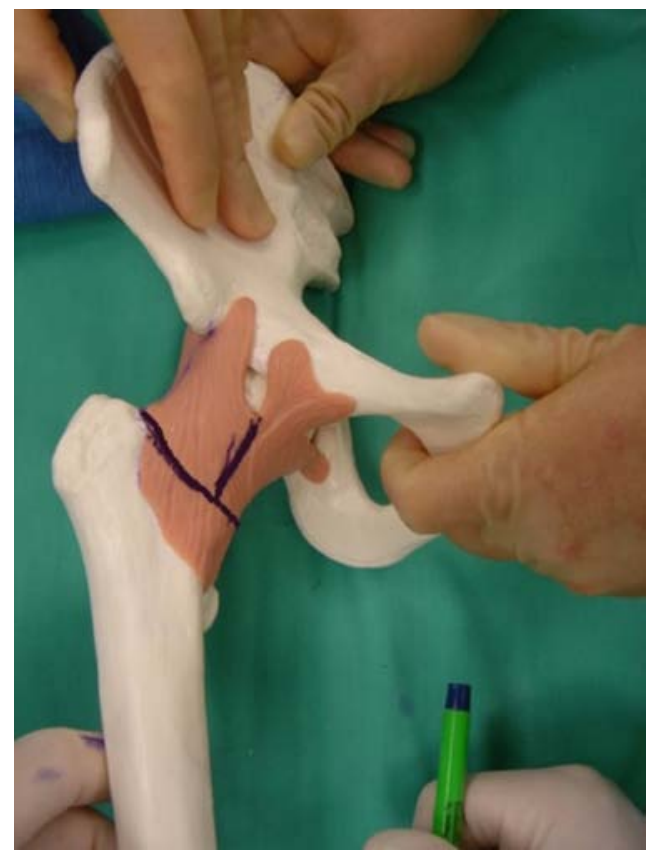

Figure 1. Planned capsular incisions performed during the DAA to THA. (Image courtesy of Kristaps $J$. Keggi, MD)

neck. The pre-planned base of the femoral neck cut is then performed, the femoral head removed, the acetabulum prepared, and the prosthetic components inserted. Depending on the exposure and mobilization needed, more, or all, of the femoral capsule can be dissected and released, yet preserved for subsequent repair. The placement of the components can be performed in a variety of ways, ranging from their insertion without any trial components, to total fluoroscopic control. Based on our surgical experience, excellent visualization of the hip and the anatomical position of the patient's spine, pelvis, and legs, we rarely use trial components other than femoral heads, but recommend them if there is any question about achieving satisfactory component position.

After the trial femoral head is placed, femoral neck length is then assessed by approximation of the hip capsule to the distal intertrochanteric line with the leg held in a neutral position, with slight flexion and internal rotation (Figure 2). If the capsule overhangs its release point on the intertrochanteric line, then the pre-operative limb/femoral neck length has been shortened. If the capsule does not reach the point where it was released, then the limb/femo-
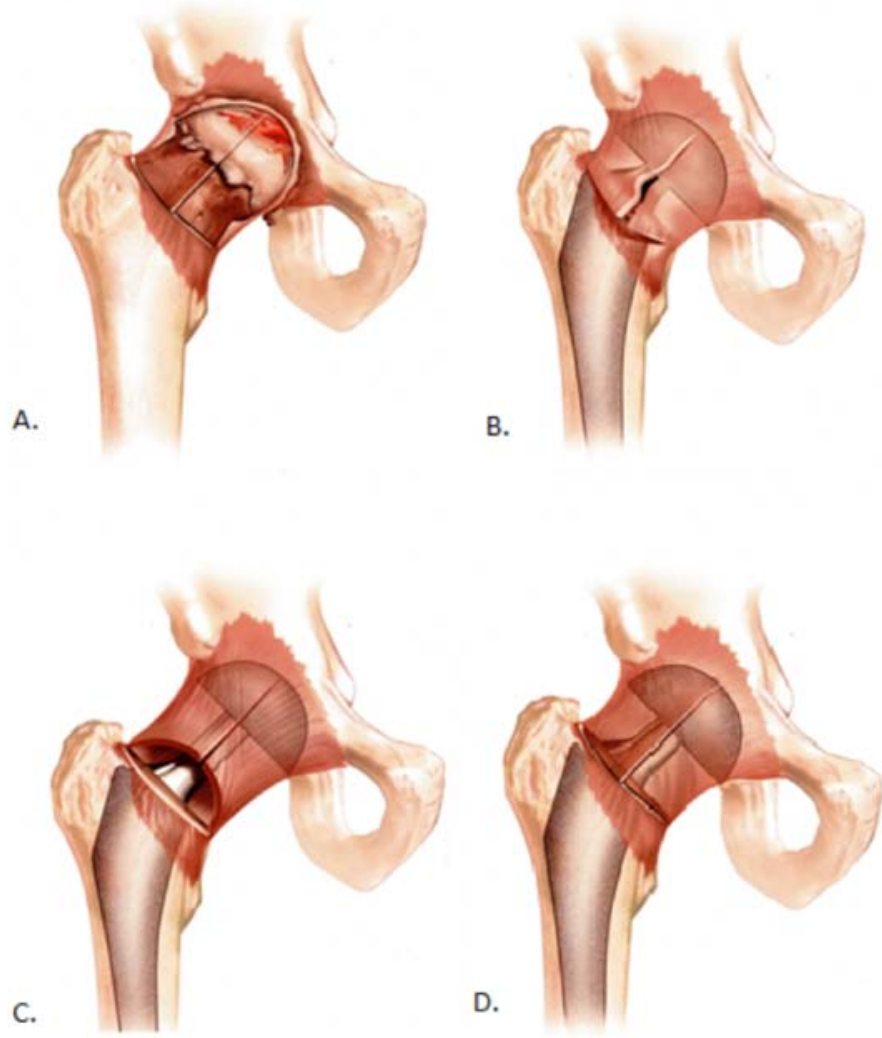

Figure 2. Illustrations demonstrating capsule position when assessing leg length. Illustration A demonstrates the native capsule position after capsulotomy. In illustration $B$, the capsule overhangs its site of release along the intertrochanteric line demonstrating that the leg is shorter than it was pre-operatively. Illustration $C$ demonstrates a gap between the proximal and distal capsular limbs, indicating the leg has been lengthened. Illustration D shows the capsule position to be unchanged. (Illustration by Genevra Garrett)

ral neck has been lengthened by the components.

Thus, a simple look at the restored position of the released capsule will allow the selection of the final femoral head to be used to achieve the leg length correction determined by the pre-operative $\mathrm{x}$-rays. Intrinsic kinematic stability of the THA is of paramount importance, and it must be tested by putting the hip through a complete arcs of motion. This can only be achieved when no traction table is used, because the leg is draped free and can be moved in all planes during the procedure to test a full range of motion and ensure there is no impingement or subluxation during the procedure prior to closure. If the joint is found to be unstable, the instability can then be corrected by an increase of the neck length by a few millimeters, or upsizing the head and liner diameter, which can be the alternative to more extreme surgical measures. 


\section{Methods}

After obtaining IRB approval to perform a medical record review, the records database was queried for primary, unilateral total hip arthroplasty (CPT 27130) performed between 2011 and 2012. A retrospective review was performed and radiographs of 80 consecutive patients with complete preoperative and postoperative images were reviewed on the radiology program PACS (Picture Archiving and Communication System). Direct capsular measurement was the primary determinant of leg length in these patients. For radiographic review, a line was drawn between the base of the teardrops and the vertical distance between this line and the lesser trochanters was measured for both the operative and nonoperative legs (Figure 3). Limb lengths were recorded in millimeters and analyzed using Microsoft Excel (Redmond, Washington, USA).

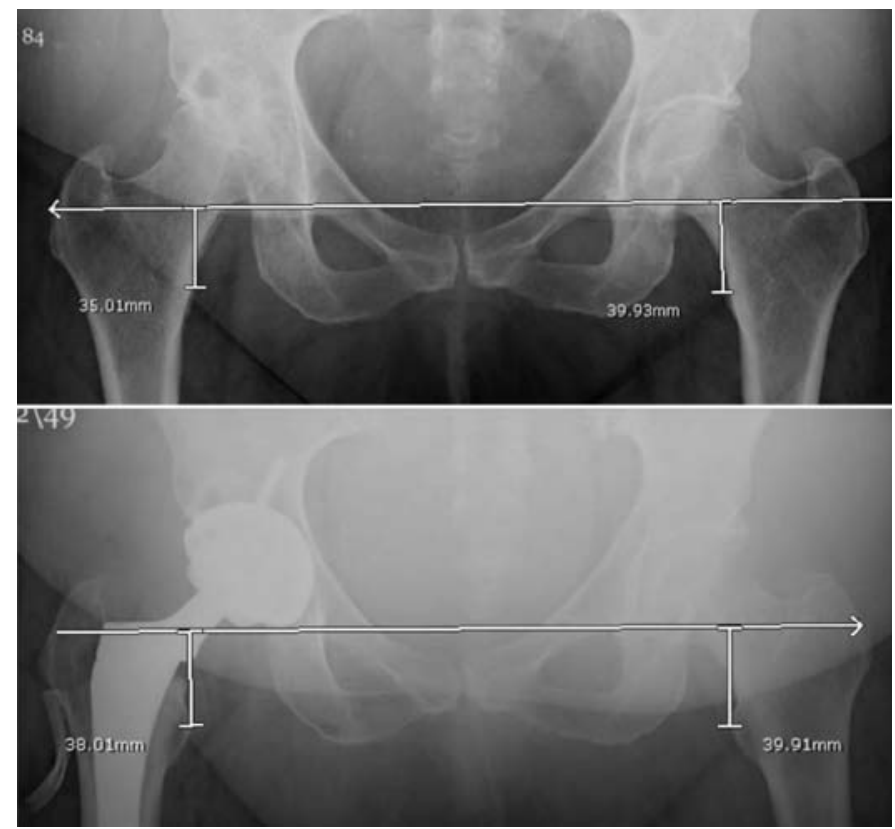

Figure 3. Patient radiographs demonstrating preoperative limb shortening due to destruction of cartilage and subchondral bone and postoperative reproduction of hip length to less than a millimeter.

Exclusion criteria included cases performed with a simultaneous bilateral technique, cases with incomplete records or radiographs, or cases with severe deformity. More specifically in cases involving major deformities, bone loss, or severe flexion contractures, we would expect a pathologically contracted, adherent, and compromised anterior capsule that is best managed with a radical capsulectomy during the procedure, precluding the use of a routine capsulotomy.

\section{Results and Discussion}

Preoperatively, many patients are shorter on the affected side due to cartilage destruction and bone remodeling secondary to the disease process. On average, our results revealed that preoperatively, the operative legs were 2.81 $+/-8.5 \mathrm{~mm}$ (SD) shorter than the nonoperative leg (range $17.7 \mathrm{~mm}$ longer to $34.1 \mathrm{~mm}$ shorter). Postoperatively, the operative legs were $1.05+/-5.64 \mathrm{~mm}$ (SD) longer than the nonoperative leg (range $14.9 \mathrm{~mm}$ longer to $13.7 \mathrm{~mm}$ shorter) (Table 1). Seven patients had a postoperative hip height discrepancy of greater than $10 \mathrm{~mm}$, however each of these patients had a similar discrepancy of over $10 \mathrm{~mm}$ preoperatively (Figure 4). Preoperative deformity, component choice, and implant position were taken into consideration for these patients in an effort to not over correct. Leg length equality was sought in every case unless it sacrificed hip stability or would alter an otherwise compensated pelvic balance in those patients with a concurrent fixed scoliotic deformity.

Table 1. Patient demographics and limb length discrepancies

\begin{tabular}{|l|l|}
\hline Male & $36(45 \%)$ \\
\hline Age (SD) & $67(+/-12.4)$ \\
\hline Left leg surgery & $32(40 \%)$ \\
\hline Preoperative limb length discrepancy (SD) & $-2.81(+/-8.50) \mathrm{mm}$ \\
\hline Postoperative limb length discrepancy (SD) & $1.05(+/-5.64) \mathrm{mm}$ \\
\hline
\end{tabular}

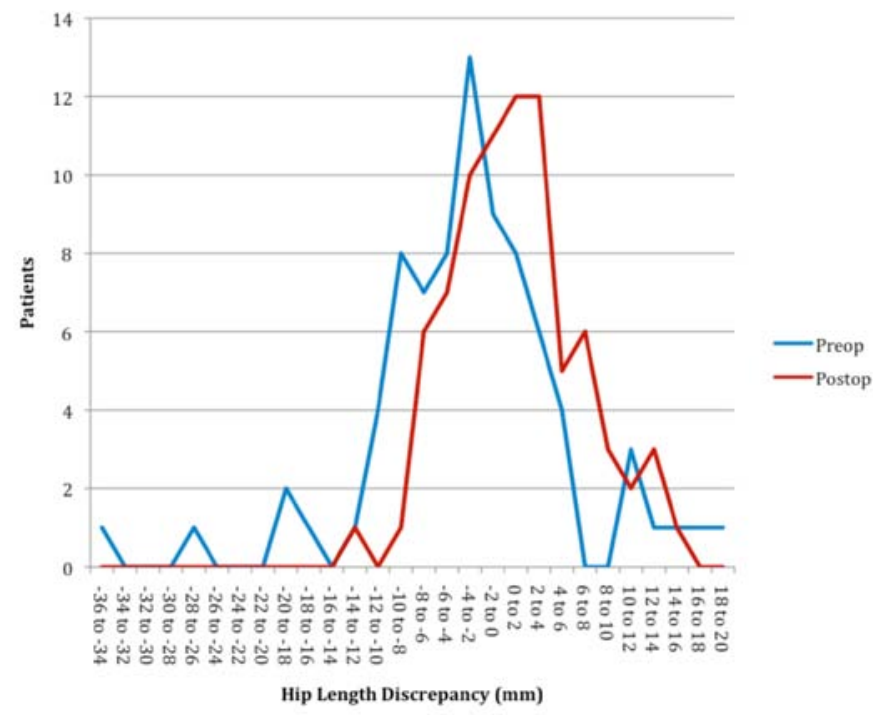

Figure 4. Frequencies of hip length discrepancies preoperatively and postoperatively.

Several novel alternative methods for assessing femoral neck length have been described including comparing the trial head and neck implants with the osteotomized femoral head using visual assessment [20]. Similar to anterior capsule assessment, this method may also be expeditious, and 
cost-effective, but may require additional equipment or specialized measurement jig. In general during our cases, a second subcapital osteotomy is created in addition to the primary basicervical osteotomy, creating a "napkin ring" cut of femoral neck bone. Thus, to measure the excised head and neck, one would need to place these together with two free saw blades to gain an accurate measurement, which is objective, but perhaps more subjectively difficult to assess than our capsular assessment as described within this paper.

A number of technical factors have been associated with leg length discrepancy, including uncemented femoral stems [21]. All patients in this evaluation underwent THA with uncemented components. The direct anterior approach for THA with a capsular sparing lends itself well to component positioning and the achievement of consistent, accurate leg length restoration.

Other potential benefits of capsular repair include increased stability and infection protection. To our knowledge, no studies have examined the stability augmented by capsular repair of THA performed through the DAA, however, Hughes et al. examined the effect of capsular repair in cadavers following hip hemiarthroplasty through a direct lateral approach and found that capsular repair required a 4-fold higher peak torque force to dislocate anteriorly [22]. Infection reduction in capsular repair has also not been directly evaluated, however, preserving additional anatomic layers might assist in microbial blockade.

In cases of major deformities, bone loss, or severe flexion contractures with compromised anterior capsules, the method we have described to "fine tune" the average hip to a few millimeters will not apply and the selection of prosthetic components and femoral heads to achieve optimum leg length and joint stability will depend on X-rays, operative findings and clinical judgment.

This paper discusses a novel technique for assessment of leg length discrepancy after THA and provides objective numerical analysis to support the accuracy of this technique at our institution. The limitations of this article include the small size of its series and that all cases were performed by a single surgeon at a single institution. Additionally, radiographs were not standardized and there may be small differences in magnification and rotation among the plain films. Due to variances in rotation of the images, we were unable to assess femoral offset which is critical to stability and abductor function, which enhance hip function after THA [23] .

Notably, this is a retrospective evaluation, and thus multiple factors aside from capsular measurement were likely used for intraoperative evaluation of leg length; most notably these included the surgeon's assessment of hip stabil- ity, shuck, and intra-operative use of the medial malleoli to measure the operative limb to the non-operative limb during the supine DAA. Future prospective evaluations comparing the various intraoperative assessments of leg length are thus necessary to determine the most accurate and reproducible method from among these options to produce reliable leg length equality. In addition, comparison of the capsular preservation technique described here to technologies such as fluoroscopy or navigation guidance would lend additional insight to this topic in the future.

\section{Disclosure}

The authors declare that there is no conflict of interest regarding the publication of this paper. For full disclosures refer to last page of this journal.

\section{References}

1. FRIBERG O. Clinical symptoms and biomechanics of lumbar spine and hip-joint in leg length inequality. Spine. 1983;8(6):643-651. doi: 10.1097/00007632-198309000-00010.

2. Mihalko W, Phillips M, Krackow K. Acute sciatic and femoral neuritis following total hip arthroplasty - A case report. Journal of Bone and Joint Surgery-American Volume. 2001;83A(4):589-592.

3. Rosler J, Perka C. The effect of anatomical positional relationships on kinetic parameters after total hip replacement. Int Orthop. 2000;24(1):23-27. doi: 10.1007/s002640050006.

4. Ranawat C. The pants too short, the leg too long! Orthopedics. 1999;22(9):845-846.

5. WOO R, MORREY B. Dislocations after total hip-arthroplasty. Journal of Bone and Joint Surgery-American Volume. 1982;64(9):1295-1306.

6. Amstutz H, Ma S, Jinnah R, Mai L. Revision of aseptic loose total hip arthroplasties. Clin Orthop. 2004(420):2-9.

7. Austin M, Hozack W, Sharkey P, Rothman R. Stability and leg length equality in total hip ar throplasty. J Arthroplasty. 2003;18(3):88-90. doi: 10.1054/arth.2003.50073.

8. Konyves A, Bannister G. The importance of leg length discrepancy after total hip arthroplasty. Journal of Bone and Joint Surgery-British Volume. 2005;87B(2):155-157. doi: $\underline{10.1302 / 0301-620 X .87 B 2.14878 .}$.

9. Wylde V, Whitehouse SL, Taylor AH, Pattison GT, Bannister GC, Blom AW. Prevalence and functional impact of patient-perceived leg length discrepancy after hip replacement. Int Orthop. 2009;33(4):905-909. doi: 10.1007/s00264-008-0563-6.

10. Upadhyay A, York S, Macaulay W, McGrory B, Robbennolt J, Bal BS. Medical malpractice in hip and knee arthroplasty. J Arthroplasty. 2007;22(6):2-7. doi: 10.1016/j.artli.2007.05.003.

11. Ng VY, Kean JR, Glassman AH. Limb-length discrepancy after hip arthroplasty. Journal of Bone and Joint Surgery-American Volume. 2013;95A(15):1426-1436. doi: 10.2106/ JBJS.L.00433.

12. Knight J, Atwater R. Preoperative planning for total hip arthroplasty: Quantitating it's utility and precision. J Arthroplasty. 1992;7:403-409.

13. Ranawat C, Rodriguez J. Functional leg-length inequality following total hip arthroplasty. J Arthroplasty. 1997;12(4):359-364. doi: 10.1016/S0883-5403(97)90190-X.

14. Charles M, Bourne R, Davey J, Greenwald A, Morrey B, Rorabeck C. Soft-tissue balancing of the hip - the role of femoral offset restoration. Journal of Bone and Joint Surgery-American Volume. 2004;86A(5):1078-1088.

15. McArthur BA, Schueler BA, Howe BM, Trousdale RT, Taunton MJ. Radiation exposure during fluoroscopic guided direct anterior approach for total hip arthroplasty. J Arthroplasty. 2015;30(9):1565-1 568. doi: 10.1016/j.arth.2015.03.029.

16. Desai A, Dramis A, Board T. Leg length discrepancy after total hip arthroplasty: A review of literature. Curr Rev Musculoskelet Med. 2013;6(4):336-341

17. Light TR, Keggi KJ. Anterior approach to hip-arthroplasty. Clin Orthop. 1980(152):255-260.

18. Kennon R, Keggi J, Zatorski L, Keggi K. Anterior approach for total hip arthroplasty: Beyond the minimally invasive technique. Journal of Bone and Joint Surgery-American Volume. 2004;86A:91-97.

19. Kennon R, Keggi J, Wetmore R, Zatorski L, Huo M, Keggi K. Total hip arthroplasty through a minimally invasive anterior surgical approach. Journal of Bone and Joint Surgery-American Volume. 2003;85A:39-48.

20. Alazzawi S, Douglas SL, Haddad FS. A novel intra-operative technique to achieve accurate leg length and femoral offset during total hip replacement. Ann R Coll Surg Engl. 2012;94(4):281-282.

21. Ahmad R, Sharma V, Sandhu H, Bishay M. Leg length discrepancy in total hip arthroplasty with the use of cemented and uncemented femoral stems. A prospective radiological study. Hip International. 2009;19(3):264-267.

22. Hughes AW, Clark D, Carlino W, Gosling O, Spencer RF. Capsule repair may reduce dislocation following hip hemiarthroplasty through a direct lateral approach. Bone \& Joint Journal. 2015;97B(1):141-144. doi: 10.1302/0301-620X.97B1.34038.

23. Liebs TR, Nasser L, Herzberg W, Ruether W, Hassenpflug J. The influence of femoral offset on health-related quality of life after total hip replacement. Bone \& Joint Journal. 2014;96B(1):36-42. 